\title{
Small Signal Modeling of Inverter-based Grid- Connected Microgrid to Determine the Zero-Pole Drift Control with Dynamic Power Sharing Controller
}

\author{
Erum Pathan \\ Department of Electrical Power Engineering, Faculty of \\ Electrical and Electronic Engineering, Universiti Tun Hussein \\ Onn Malaysia, Johor, Malaysia \\ erumasad79@gmail.com
}

\section{Usman Bashir Tayab}

School of Engineering and Built Environment, Griffith University, Nathan Campus,

Brisbane, Queensland, Australia usmanbashir.tayab@griffithuni.edu.au

\author{
Shamsul Aizam Zulkifli \\ Department of Electrical Power Engineering, Faculty of \\ Electrical and Electronic Engineering, Universiti Tun Huseein \\ Onn Malaysia, Johor, Malaysia \\ aizam@uthm.edu.my \\ Ronald Jackson \\ Department of Electrical Power Engineering, Faculty of \\ Electrical and Electronic Engineering, Universiti Tun Hussein \\ Onn Malaysia, Batu Pahat, Johor, Malaysia \\ RonaldJackNen.91@outlook.com
}

\begin{abstract}
This paper presents a small signal state space modeling of three-phase inverter-based microgrid (MG) system with consideration of improved droop control. The complete system matrices for one distribution source-grid connects to the local load have been elaborated by applying high, medium and lowfrequency clusters to the system without considering the switching action on the inverter during power-sharing. Moreover, the final matrices will be used to determine the location of the eigenvalues for the control parameters gains due to dynamic effect of the MG, by observing the root locus graph on cluster identification. Sensitivity analysis of all types of frequency cluster showed that power-sharing control parameters such as load current, source current, and inverter voltage are influencing system stability and must be considered when designing the proportional-integral (PI) control when different load scenarios have been applied from the zero-pole drifting. Those eigenvalues of the system model are indicating the frequency and damping oscillatory components when there is sudden changed at the inverter-grid connection. The matrices' eigenvalues are being plotted using MATLAB/Simulink to identify system stability region and find the PI controller parameters.
\end{abstract}

Keywords-power sharing control; microgrid; power-sharing control; small-signal stability

\section{INTRODUCTION}

Renewable energy sources are attracting more and more attention [1-2] due to the limited resevers of non-renewable resources. Power electronic converters are commonly used to interface distributed sources and grid to a microgrid (MG) which can operate either on islanded or grid-connected mode [3-5]. The small signal state space modelling of MG is the best way to observe its performance. Authors in [6-8] proposed a small signal modeling of $\mathrm{MG}$ and did not consider filter dynamics or damping filter resistance. Similarly, authors in [910] presented a natural, reference frame based, mathematical model to evaluate inverter based microgrid stability against communication time delay. Moreover, the effect of unintentional islanding stability and DC link small modeling have been developed in [11] while in [12] a small signal model of the $n$-th interconnected synchronous machine was investigated by applying the advanced control techniques which can be described the state space representation of the system. Authors in [6-12] focused on small signal modeling of MG with droop controller and did not consider power sharing response. Computational algorithms used for calculating the active and reactive power [13-15] are needed to observe the response.

In this paper, we present a small signal modeling of three phase inverter based MG using improved droop control with consideration of power-sharing response. The main contribution of this paper is the performance investigation of the proposed small signal modeling of improved droop control with consideration of low-frequency cluster stability, sensitivity of the dynamic power sharing controller gain, and effect of the line impedance parameters in different scenarios. Furthermore, by implementing this technique, the exact location and drift flow movements can be estimated in order to maintain system stability. As a model, the MG is divided into power and control blocks reconstructed into a single state space mode while transmission line, voltage source inverter, and filter are included in the power block transformation. The control block is based on dual loop controllers, i.e. inner current control and outer voltage control. In addition, it is compared with the conventional droop control to show the effectiveness of the proposed small signal model of improved droop control. 
The proposed model is verified through MATLAB simulation. Based on the simulation results, it can be concluded that transient response and poor damping have been eliminated by the use of the proposed small signal model.

\section{ISLANDED AND GRID-CONNECTED SYSTEM OVERVIEW}

Distributed renewable sources are interfaced to a MG by power converters, filters, transmission lines, loads, utilities, and points of common coupling, either in islanded or in gridconnected mode (Figure 1). Concurrently, the MG could also be operated in single or multilevel parallel inverters. This paper investigates only grid-connected mode using power-sharing technique which is based on improved droop control. Threephase voltage and current measurement signals are used to transform the time signal to a constant time signal. Calculated active and reactive powers are filtered by low pass filters and are sent to power-sharing controllers based on the $\mathrm{dq}$ components. Hence, the power-sharing controller will generate amplitude, angle and frequency references. In the islanded mode all the calculation of three-phase to direct park transforms are driven by using the reference angle from the power sharing controller.

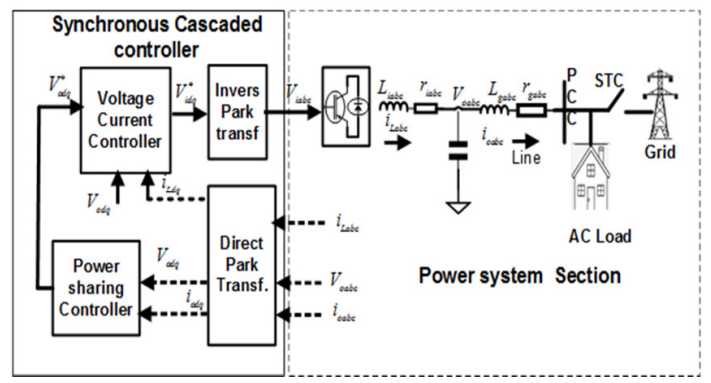

Fig. 1. Control and power system block diagram

In grid-connected mode, a phase lock loop (PLL) is used to generate the reference angle for response to the grid phase. Therefore, in a direct park transformation based PLL, the frequency is measured by forcing the $\mathrm{q}$ axis component of a voltage to zero. Cascaded controllers for controlling the inner current and outer voltage control of the system are needed to record the dynamic behavior of the load changes. The voltage reference for the voltage controller is obtained from the frequency and amplitude signals by the power controller. The overall system dynamics including power, voltage, current controller, inverter, and line impedance are given in state space based small signal modeling section when it is in gridconnected mode. If the system is used in islanded mode, load parameter dynamics can also be considered.

\section{STATE SPACE-BASED SMALL SIGNAL MODEL}

The MG model needs to be linearized around the operating point by the use of small signal techniques in order to study the dynamics of power and control system. In this section, the mathematical model of the system is discussed in detail.

\section{A. Dynamics of Synchronous Voltage, Current Control, and LCL Filter}

The dynamic model equations start when the inverter controller is modeled based on dual loops performance. The PI controller is used to get zero steady-state error. In (1), $\varphi_{d q}$ is the error between the measured and set point voltage. This error is sent to the voltage controller for tracking the reference signal and generating input reference signal for current controller [16]. The algebraic dynamic of voltage control is given as:

$$
\begin{gathered}
\stackrel{*}{i_{l d}}=-\omega_{o} C_{f} V_{o q}+k_{p v}\left(V_{o d}^{*}-V_{o d}\right)+k_{i v} \varphi_{d} \\
i_{l q}^{*}=+\omega_{o} C_{f} V_{o d}+k_{p v}\left(V_{o q}^{*}-V_{o q}\right)+k_{i v} \varphi_{q}
\end{gathered}
$$

where $i_{l d}^{*}$ and $i_{l q}^{*}$ are the inputs for current controller, $K_{p v}$ and $K_{i v}$ are the PI regulator gains.

The current controller ensures that the inverter output voltage follows the reference signal which is coming from the output of the voltage inverter. It produces the difference between measured and reference filter inductor current and produces measured voltages. In addition, it eliminates the cross-coupling terms by using the dq format to determine the signal reference for the generation of pulse width modulation (PWM) for the inverter switching. As for the inner loop control, the voltages are given in (2):

$$
\begin{gathered}
V_{i d}^{*}=-\omega_{o} L_{i} i_{l q}+k_{p c}\left(i_{l d}^{*}-i_{l d}\right)+k_{i c} \gamma_{d} \\
V_{i q}^{*}=+\omega_{o} L_{i f} i_{l d}+k_{p c}\left(i_{l q}^{*}-i_{l q}\right)+k_{i c} \gamma_{q}
\end{gathered}
$$

where $V_{l d}^{*}$ and $V_{l q}^{*}$ are the measured signal, $K_{p c}$ and $K_{i c}$ are the PI regulator gains.

\section{B. Power-Sharing and Proposed Power-Sharing Controller}

Instantaneous active and reactive inverter power are calculated by using the direct and quadratic axis-based inverter output current $i_{o d q}$ and voltage $v_{o d q}$ of the system [17]. In order to cancel high-frequency components and ripples, the instantaneous power is passed through a low pass filter to obtain the average power of the system which is given in (3), where $\omega_{c}$ is the cut off frequency:

$$
\begin{aligned}
& P_{i n v}=\frac{\omega_{c}}{s+\omega_{c}} \times 1.5\left(V_{o d} i_{o d}+V_{o q} i_{o q}\right) \\
& Q_{i n v}=\frac{\omega_{c}}{s+\omega_{c}} \times 1.5\left(V_{o d} i_{o q}-V_{o q} i_{o d}\right)
\end{aligned}
$$

In the islanded mode the inverter does not use any reference as external signal but only the current controller. Hence, the inverter output frequency and voltage can be adjusted by applying droop theory mechanism for power sharing. The characteristics equations of droop controller are given in (4) as for grid-connected mode.

$$
\begin{aligned}
& \omega=\omega_{o}-m\left(P_{i n v}-P_{r e f}\right) \\
& V_{o d}{ }^{*}=V_{o}-n\left(Q_{i n v}-Q_{r e f}\right), \Delta V_{o q}^{*}=0
\end{aligned}
$$

By considering $P_{r e f}$ and $Q_{r e f}$ are equal to zero in islanded mode, so is the droop. Hence, the phase angle of the inverter is 
set by taking the integral of frequency $\dot{\delta}=-m P_{i n v}$. The proportional frequency droop coefficient $m$ and the proportional voltage droop coefficient $n$ are calculated by:

$$
m=\frac{\omega_{o}-\omega}{P_{i n v}}, n=\frac{V_{o}-V_{o d}^{*}}{Q_{i n v}}
$$

The complete dynamic linear small signal state space model of the inverter in grid-connected mode can be obtained by combining all equations of power controller, voltage controller, current controller, LCL filter, line, and bus:

$$
\left.\Delta \dot{X}_{\text {inv }}\right]\left[\begin{array}{ccccc}
a & 0 & 0 & B_{P} & 0 \\
b & 0 & 0 & B_{v 2} & 0 \\
c & B_{C 1} C_{v} & 0 & d & 0 \\
e & f & g & h & B_{L C L 2} \\
B_{b u s} & 0 & 0 & 0 & 0
\end{array}\right]_{14 \times 14}\left[\begin{array}{c}
\Delta \delta \\
\Delta P_{i v v} \\
\bullet \\
\bullet \\
\dot{\bullet} \\
\Delta \delta_{\text {odq }}
\end{array}\right]_{1 \times 14}
$$

where

$$
\begin{aligned}
& a=A_{P}+B_{P_{-} c o m} C_{P_{w}}, b=B_{v 1} C_{p v} \\
& c=B_{v 1} D_{v 1} C_{p v}, d=B_{C 1} D_{v 2}+B_{C 2} \\
& e=B_{L C L 1} D_{C 1} D_{v 1} C_{p v}+B_{L C L 3} C_{p w} \\
& f=B_{L C L 1} D_{C 1} C_{v}, g=B_{L C L 1} C_{C} \\
& h=A_{L C L}+B_{L C L 1}\left(D_{C 1} D_{v 2}+D_{C 2}\right)
\end{aligned}
$$

In order to improve the system transient response and reduce the system oscillation, the differential of power in conventional power-sharing control should be added. The proposed power sharing control system is:

$$
\begin{aligned}
& \omega=\omega_{o}-m\left(P_{i n v}-P_{r e f}\right)-m_{d} \frac{d P_{i n v}}{d t} \\
& V_{o d}=V_{o}-n\left(Q_{i n v}-Q_{r e f}\right)-n_{d} \frac{d Q_{i n v}}{d t}
\end{aligned}
$$

Consequently, the complete inverter model can be modified as per the dynamic power sharing controller and the proposed bus model is given in (8):

$$
\left.\Delta X_{i n v d y n}^{\bullet}\right]_{a_{1}}\left[\begin{array}{ccccc}
a_{1} & 0 & 0 & a_{11} & 0 \\
b_{1} & 0 & 0 & b_{11} & 0 \\
c_{1} & c_{11} & 0 & c_{12} & 0 \\
d_{1} & d_{11} & d_{12} & d_{13} & d_{14} \\
e_{1} & 0 & 0 & 0 & 0
\end{array}\right]_{14 \times 14}\left[\begin{array}{c}
\Delta \delta \\
\Delta P_{i n v} \\
\bullet \\
\bullet \\
\Delta i_{o d q} \\
\Delta \delta_{2}
\end{array}\right]_{1 \times 14}
$$

where:

$$
\begin{aligned}
& a_{1}=A_{P}^{\text {dynamic }}+B_{p c o m}^{\text {dynamic }} C_{P w}^{\text {dynamic }}, a_{11}=B_{P}^{\text {dynamic }} \\
& b_{1}=B_{v 1} C_{p v^{\text {dymanic }}}, b_{11}=B_{v 2}+B_{v 1} D_{p v}^{\text {dynamic }} \\
& c_{1}=B_{v 1} D_{v 1} C_{p v^{d \text { dynamic }}}, c_{11}=B_{C 1} C_{v}, e_{1}=B_{p c c}^{\text {dynamic }} \\
& c_{12}=B_{C 1} D_{v 2} D_{p v}^{\text {dynamic }}+B_{c 1} D_{v 2}+B_{C 2} \\
& d_{1}=B_{L C L 1} D_{C 1} D_{v 1} C_{p v}^{\text {dynamic }}+B_{L C L 3} C_{p w}^{\text {dynamic }} \\
& d_{11}=B_{L C L 1} D_{C 1} C_{v}, d_{12}=B_{L C L 1} C_{C}, d_{14}=B_{L C L 2} \\
& d_{13}=A_{L C L}+B_{L C L 1}\left(D_{C 1} D_{v 2}+D_{C 2}\right)+B_{L C L 1} D_{C 1} D_{v 1} D_{p v}^{\text {dynamic }}+B_{L C L 3} D_{p w}^{\text {dynamic }}
\end{aligned}
$$

\section{STATE SPACE BASED PROPOSED SYSTEM MODEL}

The previous section is based on the complete system model of MG as illustrated in Figure 1, which is based on 14 state variables. However, the reduced system model is only based on 5 state variables that are mostly effects on powersharing accuracy and stability.

\section{A. Reduced Model of Power-Sharing Controller}

The reduced matrixes model can be taken into account using low frequency-based system parameters consisting of line impedance, power-sharing controller and filter only. Compared to medium and high frequency-based system model, the filter and cascaded controller parameters would not be affected by the stability when the sharing is happening to the inverter.

$$
\dot{i_{o d q}}=\frac{-r_{g}}{L_{g}} i_{o d q} \pm \omega_{o} i_{o q d}-\frac{1}{L_{g}} V_{o d q}+\frac{1}{L_{g}} V_{p c c_{-} d q}
$$

The reduced system model based on the low-frequency system parameters and line impedance is given in (10):

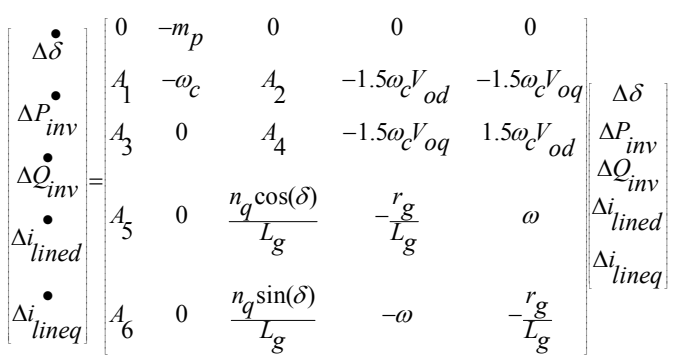

where:

$$
\begin{aligned}
& A_{1}=1.5 \omega_{c}\left(i_{\text {lined }}\left(-V_{\text {od }} \sin (\delta)-V_{\text {oq }} \operatorname{Cos}(\delta)\right)-i_{\text {lineq }}\left(V_{\text {oq }} \operatorname{Cos}(\delta)-V_{\text {od }} \sin (\delta)\right)\right. \\
& A_{2}=1.5 \omega_{c} n_{q}\left(-i_{\text {lined }} \operatorname{Cos}(\delta)-i_{\text {lineq }} \sin (\delta)\right) \\
& A_{3}=1.5 \omega_{c}\left(i_{\text {lineq }}\left(-V_{\text {od }} \sin (\delta)-V_{\text {oq }} \operatorname{Cos}(\delta)\right)-i_{\text {lined }}\left(V_{\text {od }} \operatorname{Cos}(\delta)-V_{q d} \sin (\delta)\right)\right. \\
& A_{4}=-\omega_{c}+1.5 \omega_{c} n_{q}\left(-i_{\text {lineq }} \operatorname{Cos}(\delta)+i_{\text {lined }} \sin (\delta)\right) \\
& A_{6}=-\frac{\left(V_{\text {od }} \operatorname{Cos}(\delta)-V_{\text {oq }} \sin (\delta)\right)}{L_{g}}, A_{5}=-\frac{\left(-V_{\text {od }} \sin (\delta)-V_{\text {oq }} \operatorname{Cos}(\delta)\right)}{L_{g}}
\end{aligned}
$$

\section{B. Proposed Power Sharing Controller}

A reduced system model of dynamic power sharing controller is based on only 5 out of 14 states of the system because the reduced model is only based on the line impedance effect and dynamic power sharing controller parameters. The reduced state space matrix is given below: 


$$
\left[\begin{array}{c}
\Delta \dot{\delta} \\
\dot{P_{i n v}} \\
\Delta{\dot{Q_{i n v}}}_{\dot{\cdot}} \\
\Delta \dot{i}_{\text {lined }} \\
\Delta \dot{i_{\text {lineq }}}
\end{array}\right]=\left[\begin{array}{ccccc}
A_{1} & A_{2} & A_{3} & A_{4} & A_{5} \\
A_{6} & -\omega_{c} & A_{8} & A_{9} & A_{10} \\
A_{11} & 0 & A_{13} & A_{14} & A_{15} \\
A_{16} & 0 & A_{18} & -\frac{R_{g}}{L_{g}} & \omega_{o} \\
A_{21} & 0 & A_{23} & \omega_{o} & -\frac{R_{g}}{L_{g}}
\end{array}\right]_{5 \times 5}\left[\begin{array}{c}
\Delta \delta \\
\Delta P_{i n v} \\
\Delta Q_{i n v} \\
\Delta i_{\text {lined }} \\
\Delta i_{\text {lineq }}
\end{array}\right]_{1 \times 5}
$$

where:

$$
\begin{aligned}
& A_{1}=A_{3}=1.5 \omega_{c} m_{d}\left(-i_{\text {lined }}\left(-V_{\text {od }} \sin (\delta)-V_{\text {oq }} \operatorname{Cos}(\delta)\right)-i_{\text {lineq }}\left(V_{\text {oq }} \operatorname{Cos}(\delta)-V_{\text {od }} \sin (\delta)\right)\right. \\
& A_{2}=-m+m_{d} \omega_{c}, A_{4}=-1.5 m_{d} \omega_{c} V_{o q}, A_{5}=-1.5 m_{d} \omega_{c} V_{o d}, A_{9}=1.5 \omega_{c} V_{o d}, A_{10}=1.5 \omega_{c} V_{o q} \\
& A_{6}=1.5 \omega_{c}\left(i_{\text {lined }}\left(-V_{\text {od }} \sin (\delta)-V_{\text {oq }} \operatorname{Cos}(\delta)\right)+i_{\text {lineq }}\left(V_{\text {oq }} \operatorname{Cos}(\delta)-V_{\text {od }} \sin (\delta)\right)\right. \\
& \left.\left.A_{8}=1.5 \omega_{c}\left(i_{\text {lined }}\left(-n+\omega_{c} n_{d}\right) \operatorname{Cos}(\delta)\right)+i_{\text {lineq }}\left(-n+\omega_{c} n_{d}\right) \sin (\delta)\right)\right), A_{14}=-1.5 \omega_{c} V_{\text {oq }} \\
& A_{11}=1.5 \omega_{c}\left(-i_{\text {lined }}\left(-V_{\text {od }} \sin (\delta)-V_{\text {oq }} \operatorname{Cos}(\delta)\right)-i_{\text {lineq }}\left(V_{\text {oq }} \operatorname{Cos}(\delta)-V_{\text {od }} \sin (\delta)\right)\right. \\
& \left.\left.A_{13}=-\omega_{c}+1.5 \omega_{c}\left(i_{\text {lined }}\left(-n+\omega_{c} n_{d}\right) \operatorname{Cos}(\delta)\right)-i_{\text {lineq }}\left(-n+\omega_{c} n_{d}\right) \sin (\delta)\right)\right) \\
& A_{15}=1.5 \omega_{c} V_{o d}, A_{16}=-\frac{\left(-V_{o d} \sin (\delta)-V_{o q} \operatorname{Cos}(\delta)\right)}{L_{g}}, A_{18}=-\frac{\left.\left(-n+\omega_{c} n_{d}\right) \operatorname{Cos}(\delta)\right)}{L_{g}} \\
& A_{21}=-\frac{\left(V_{o d} \operatorname{Cos}(\delta)-V_{o q} \operatorname{Sin}(\delta)\right)}{L_{g}}, A_{23}=A_{28}=-\frac{\left.\left(-n+\omega_{c} n_{d}\right) \operatorname{Sin}(\delta)\right)}{L_{g}}
\end{aligned}
$$

\section{RESULTS AND DISCUSSION}

The small signal modeling of the MG system given in Figure 1 has been tested and evaluated using the eigenvalues technique applied to Matlab/Simulink. The eigenvalues are found from (6) and (8) with a specific PI gain value technique that observes transient behavior, damping and stability of the system. The control system plant will be stable if the poles of the characteristic equations lie on the left half plan. When the dynamic controller has been applied, an imaginary complex conjugate of the characteristic equation is exhibited on the oscillatory behavior of the dynamic system and a more transient oscillation can be observed. On the other hand, if the imaginary eigenvalues are going away from the left half plan a faster transient and reduced rise time are disclosed.

\section{A. Stability Test of Complete and Reduced System Model Parameters Effect.}

The individual small signal model by its differential equations of LCL filter, line impedance, cascaded current, voltage, and power-sharing controller, is embedded in order to study the dynamic behavior of the power-sharing mechanism of the MG. This approach is also used to analyze the stability of the system. Table I shows the parameters of the initial condition of the system. The wide band of dynamic modes of the power-sharing mechanism of the MG system can be seen in Figure 2. The frequency scale separation between different modes of different control loops can be observed in Figure 3. It should be noted that timescale separation is crucial for designing a typical reduced power system. The filters and current controllers are mainly designed with high-frequency modes. The switching frequency is mainly chosen as per the inverter's rating and of switching losses. The inner current controller bandwidth should be higher than the resonance frequency of the passive filter and 4 to 10 times less than the switching frequency of the inverter. Moreover, the LCL filters resonance frequency should be half times less than the switching frequency and 10 times greater than the nominal frequency. Cluster 3 from Figure 2 illustrates the dynamic behavior of the inner controller of the power-sharing mechanism of the MG.

TABLE I. INITIAL CONDITION SYSTEM PARAMETERS

\begin{tabular}{|c|c|c|c|}
\hline Parameters & Values & Parameters & Values \\
\hline$r_{i}$ & $0.1 \Omega$ & $\delta_{2}$ & 2 \\
\hline$L_{i}$ & $1.8 \mathrm{e}-3 \mathrm{H}$ & $m$ & $1 \mathrm{e}-5$ \\
\hline$r_{g}$ & $0.23 \Omega$ & $n$ & $1 \mathrm{e}-5$ \\
\hline$L_{g}$ & $0.1 \mathrm{H}$ & $m_{d}$ & $2 \mathrm{e}-6$ \\
\hline$C_{f}$ & $25 \mathrm{e}-6 \mathrm{~F}$ & $n_{d}$ & $2 \mathrm{e}-6$ \\
\hline$V_{\text {rated }}$ & $311 \mathrm{~V}$ & $k_{p c}$ & 5.3 \\
\hline$P_{\text {rated }}$ & $2.2 \mathrm{KW}$ & $k_{i c}$ & 0 \\
\hline$P_{\text {load }}$ & $726 \mathrm{~W}$ & $k_{i v}$ & 100 \\
\hline$\omega$ & $31.415 \mathrm{rad} / \mathrm{s}$ & $k_{p v}$ & 0.04 \\
\hline$\omega_{c}$ & $62.831 \mathrm{rad} / \mathrm{s}$ & $\delta$ & 0 \\
\hline
\end{tabular}

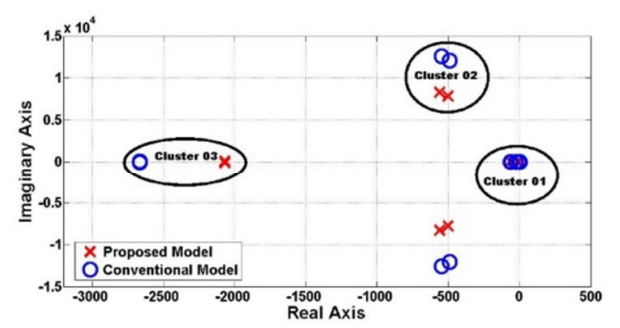

Fig. 2. Eigenvalues of dynamic power sharing and power sharing based complete signal model $(\mathrm{x}=$ proposed, $\mathrm{o}=$ conventional model $)$

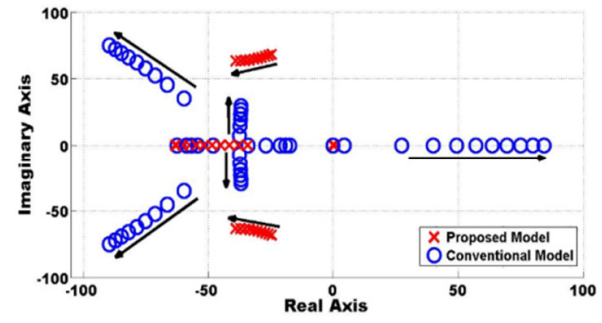

Fig. 3. Effect of proportional droop coefficients $m$ and $n$ on dynamic power sharing controller and power-sharing controller ( $\mathrm{x}=$ proposed, $\mathrm{o}=$ conventional model)

Cluster 2 from Figure 2 illustrates the dynamic effect of voltage controller which is designed on medium bandwidth and based on medium frequency modes. The voltage controller bandwidth should be 5 to 10 times less than the inner current controller in order to keep monitoring the tracking resolution and the stability of the inner control loop. Outer control loop dynamics are illustrated by cluster 1 in Figure 2 which is based on power-sharing controller and low pass filters. This control loop is mainly designed with very low bandwidth, since the average outer power-sharing controller in grid-connected mode needs gradual change in the reference voltage. Average power is extracted from the synchronous reference frame based on the instantaneous power components and low pass filters with low cut-off frequencies. Consequently, this controller bandwidth should be lower than voltage controller bandwidth. 


\section{B. The Effect of Power-Sharing Co-Efficient and Dynamic Power-Sharing Coefficient}

The eigenvalues of the power-sharing controller are illustrated in Figure 3, which presents how the power-sharing coefficient controller effects on system stability. Figure 4 shows the dynamic power sharing controller with the effect of the power-sharing coefficient. In Figure 3, when the active power gain is varied from $1 \mathrm{e}-5$ to $5 \mathrm{e}-4$, the dynamic power sharing controller real poles are moving towards the left side whereas the real poles of power-sharing controller are moving towards the right side. Similarly, the dynamic power sharing controller-based system is said to be stable but the powersharing controller-based system leads towards instability and system response becomes more oscillatory. When the active dynamic power sharing gain is increased, the dominant poles of the imaginary axis are varying and the system becomes faster. The effect of active dynamic power sharing gain is illustrated in Figure 4.

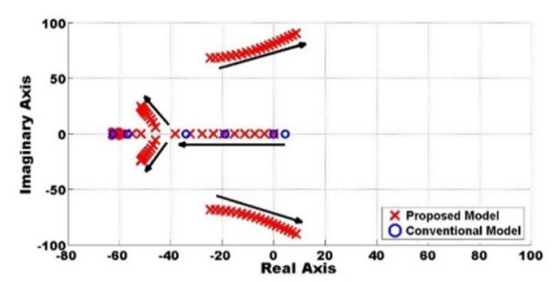

Fig. 4. Effect of dynamic active power droop gain md on dynamic power sharing controller and power-sharing controller $(\mathrm{x}=$ proposed, $\mathrm{o}=$ conventional model).

\section{Effect of Line Impedance Parameters}

The line impedance parameters also affect the system stability of the dynamic power sharing controller as well as the power sharing controller-based system. The effect of the line impedance variation is illustrated in Figures 5-6.

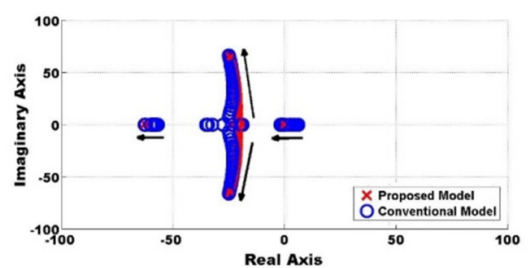

Fig. 5. Effect of line impedance $L g$ is varying and $R g$ is fixed (x=proposed, $\mathrm{o}=$ conventional model)

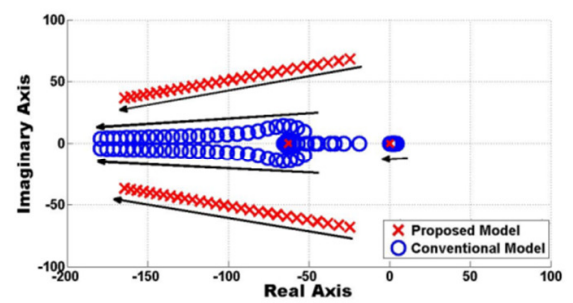

Fig. 6. Effect of line impedance $(\mathrm{x}=$ proposed, $\mathrm{o}=$ conventional model $)$
When the inductance of line impedance is increased, the system never becomes unstable, but the large values of the inductive part could not be the real line impedance values. Hence, the $X g / R g$ is directly affected on system dynamics, so the leading of inductance of the line impedance reduces the steady state error while the system transient response for increasing value of the resistive part of line impedance is damped.

\section{Validation by MATLAB/Simulink Sim Power Tool}

Power-sharing controller and dynamic power sharing controller effect were validated by MATLAB/Simulink. The set point of active power was considered $726 \mathrm{~W}$. Poor damping, slow transient response, and maximum by using the conventional power-sharing controller overshoot can be seen in Figure 7. Poor damping and transient have been improved by using the dynamic power sharing control without compromising the steady-state performance.

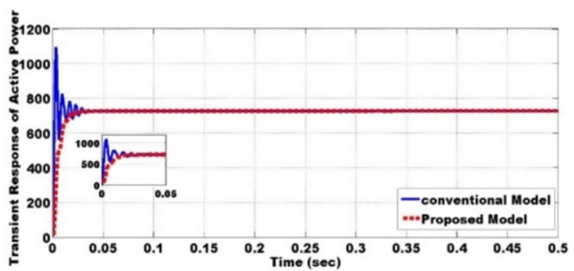

Fig. 7. Transient active power of conventional and proposed model.

\section{CONCLUSION}

A small signal modeling of high-frequency cluster, medium frequency cluster and low-frequency cluster based $\mathrm{MG}$ for dynamic power control is presented in this paper. All the submodel parts have been combined on common reference frame in order to get the complete model of the MG. Analyzation of sensitivity and stability of system parameters is based on the eigenvalues technique of the developed small signal state space model and the reduced small signal state space model in different scenarios. It is concluded that power-sharing and dynamic power sharing based low-frequency mode are highly sensitive in a network of MGs. Cascaded inverter controller, loads and network dynamic sensitivity is based on the highfrequency cluster. Low-frequency cluster stability and sensitivity of the active power sharing and of the proposed dynamic power sharing controller gain, and the effect of the line impedance parameters have been investigated in different scenarios. It can be concluded that transient response and poor damping have been improved by the proposed dynamic power sharing controller.

\section{ACKNOWLEDGEMENT}

Authors gratefully acknowledge the support of Universiti Tun Hussein Onn Malaysia (UTHM), Advanced Control on Power Converters (ACPC) group, FKEE, and Research University Grant Program (U998), UTHM for undertaking this research activity. 


\section{REFERENCES}

[1] F. Blaabjerg, R. Teodorescu, M. Liserre, A. V Timbus, "Overview of Control and Grid Synchronization for Distributed Power Generation Systems", IEEE Transactions on Industrial Electronics, Vol. 53, No. 5, pp. 1398-1409, 2006

[2] A. Vidal, F. D. Freijedo, A. G. Yepes, P. Fernandez-Comesana, J. Malvar, O. Lopez, J. Doval-Gandoy, "Assessment and optimization of the transient response of proportional-resonant current controllers for distributed power generation systems", IEEE Transactions on Industrial Electronics, Vol. 60, No. 4, pp. 1367-1383, 2013

[3] IEEE Standards Coordinating Committee, 1547.4-2011-IEEE Guide for Design, Operation, and Integration of Distributed Resource Island Systems with Electric Power Systems, IEEE, 2011

[4] Y. Han, M. Luo, X. Zhao, J. M. Guerrero, L. Xu, "Comparative Performance Evaluation of Orthogonal-Signal-Generators-Based SinglePhase PLL Algorithms - A Survey", IEEE Transactions on Power Electronics, Vol. 31, No. 5, pp. 3932-3944, 2016

[5] P. Shen, Y. Han, C. Lu, J. M. Guerrero, "Small-signal modeling of the PVR-based AD scheme and controller design for three-phase standalone DG system", Journal of Electrical Engineering \& Technology, Vol. 11, No. 5, pp. 1165-1178, 2016

[6] F. Katiraei, M. R. Iravani, P. W. Lehn, "Small-signal dynamic model of a micro-grid including conventional and electronically interfaced distributed resources", IET Generation, Transmission \& Distribution, Vol. 1, No. 3, pp. 369-378, 2007

[7] N. Pogaku, M. Prodanovic, T. C. Green, "Modeling, analysis and testing of autonomous operation of an inverter-based microgrid", IEEE Transactions on Power Electronics, Vol. 22, No. 2, pp. 613-625, 2007

[8] M. Rasheduzzaman, J. Mueller, J. W. Kimball, "Small-signal modeling of a three-phase isolated inverter with both voltage and frequency droop control", 2014 IEEE Applied Power Electronics Conference and Exposition, Fort Worth, USA, March 16-20, 2014

[9] W. R. Issa, M. A. Abusara, S. M. Sharkh, "Control of Transient Power during Unintentional Islanding of Microgrids", IEEE Transactions on Power Electronics, Vol. 30, No. 8, pp. 4573-4584, 2015

[10] U. B. Tayab, M. Azrik, B. Roslan, L. J. Hwai, M. Kashif, "A review of droop control techniques for microgrid", Renewable and Sustainable Energy Reviews, Vol. 76, pp. 717-727, 2017

[11] W. Issa, M. Abusara, S. Sharkh, T. Mallick, "A small signal model of an inverter-based microgrid including DC link voltages", 17th European Conference on Power Electronics and Applications, Geneva, Switzerland, September 8-10, 2015

[12] U. B. Tayab, Q. M. Humayun, "Enhanced droop controller for operating parallel-connected distributed-generation inverters in a microgrid", Journal of Renewable and Sustainable Energy, Vol. 10, No. 4, 2018

[13] U. B. Tayab, M. Kashif, "A modified droop controller for parallel operation of single-phase inverters in Islanded microgrid", International Journal of Intelligent Engineering \& Systems, Vol. 10, No. 4, pp. 11-17, 2017

[14] R. S. Herrera, P. Salmeron, J. R. Vazquez, S. P. Litran, A. Perez, "Generalized instantaneous reactive power theory in poly-phase power systems", 13th European Conference on Power Electronics and Applications, Barcelona, Spain, September 8-10, 2009

[15] A. Tuladhar, H. Jin, T. Unger, K. Mauch, "Control of parallel inverters in distributed AC power systems with consideration of line impedance effect”, IEEE Transactions on Industrial Applications, Vol. 36, No. 1, pp. 131-138, 2000

[16] B. Zhang, X. Yan, D. Li, X. Zhang, J. Han, X. Xiao, "Stable Operation and Small-Signal Analysis of Multiple Parallel DG Inverters Based on a Virtual Synchronous Generator Scheme", Energies, Vol. 11, No. 1, pp. $1-22,2018$

[17] K. Hashmi, M. M. Khan, S. Habib, H. Tang, "An Improved Control Scheme for Power Sharing between Distributed Power Converters in Islanded AC Microgrids", International Conference on Frontiers of Information Technology, Islamabad, Pakistan, December 8-20, 2017 